\title{
An Unusual Mimicker of a Pancreatic Pseudocyst
}

\author{
Sang Ah Choi, Chang-II Kwon, Gwangil Kim, Daejung Kim and Sung Hoon Choi \\ Digestive Disease Center, CHA Bundang Medical Center, CHA University, Seongnam, Korea
}

A 46-year-old woman without a significant history presented our emergency room with acute epigastric pain. Abdominal computed tomography (CT) and magnetic resonance cholangiopancreatography (MRCP) demonstrated a well-defined 2.1-cm cystic lesion at the neck of the pancreas, without peripancreatic infiltration, that was connected to the pancreatic duct (Fig. 1A). Serum laboratory findings were as follows: amylase $142 \mathrm{U} / \mathrm{L}$, lipase $149 \mathrm{U} / \mathrm{L}$, carcinoembryonic antigen (CEA) $0.94 \mathrm{ng} / \mathrm{mL}$, and cancer antigen 19-9 10.22 U/mL. Endoscopic ultrasound (EUS) demonstrated a distinct anechoic lesion connected to the main pancreatic duct (Fig. 1B). EUS-guided fine needle aspiration (EUS-FNA) retrieved clear cystic fluid with low viscosity. Histopathologic examination of the cystic fluid demonstrated ductal epithelial cells without a malignant component. Analysis of the cystic fluid showed CEA $10.45 \mathrm{ng} /$ $\mathrm{mL}$ and amylase $126 \mathrm{IU} / \mathrm{L}$, suggesting low potential for malignancy. Thus, the pancreatic cystic lesion was initially considered a pseudocyst. Follow-up CT performed after 3 months revealed a short stricture of the pancreatic duct with mild upstream dilatation around the cystic lesion. Endoscopic retrograde cholangiopancreatography demonstrated a connection of the cystic lesion (arrow) to the main pancreatic duct (Fig. 1C); a plastic stent was inserted to improve the stricture. During the 6-month follow-up period after initial presentation, the size of the cystic lesion did not dramatically decrease (Fig. 1D). Thereafter, the patient underwent central pancreatectomy. Histo-

Received: October 12, 2017 Revised: December 14, 2017

Accepted: December 15, 2017

Correspondence: Chang-Il Kwon

Digestive Disease Center, CHA Bundang Medical Center, CHA University, 59 Yatap-ro, Bundang-gu, Seongnam 13496, Korea

Tel: +82-31-780-5641, Fax: +82-31-780-5219, E-mail: endoscopy@cha.ac.kr

ORCID: https://orcid.org/0000-0003-3621-9023

cc This is an Open Access article distributed under the terms of the Creative Commons Attribution Non-Commercial License (http://creativecommons.org/ licenses/by-nc/3.0) which permits unrestricted non-commercial use, distribution, and reproduction in any medium, provided the original work is properly cited. pathologic examination demonstrated a unilocular cyst containing serosanguineous fluid, and a surrounding solid component that was diagnosed as a Grade 2 pancreatic neuroendocrine tumor (pNET) (Fig. 2). Immunohistochemical staining showed positive results for CK, CD56, and synaptophysin.

Pancreatic cystic lesion is a common incidental finding in clinical practice. CT, EUS-FNA, and MRCP are helpful for the differential diagnosis of pancreatic cystic lesion. However, if the diagnosis is uncertain, the clinical experience and decision of the treating physician could also be an important diagnostic tool.

Although pNETs are typically solid, approximately 10\%$20 \%$ of them undergo cystic degeneration, resulting in the radiologic interpretation of a pseudocyst. ${ }^{1}$ CT, MRCP, EUS, and EUS-FNA with cystic fluid analysis aid the diagnosis of cystic pNETs. $^{2}$ Although cross-sectional images and cystic fluid markers are strongly suggestive of a benign pseudocyst, a possibility of malignancy should be considered throughout. Surgical resection of primary pNETs is associated with improved survival for patients in all stages of cancer, and it should be considered in suitable candidates. ${ }^{3}$

Conflicts of Interest

The authors have no financial conflicts of interest.

\section{REFERENCES}

1. Kawamoto S, Johnson PT, Shi C, et al. Pancreatic neuroendocrine tumor with cystlike changes: evaluation with MDCT. AJR Am J Roentgenol 2013;200:W283-W290.

2. Brugge WR, Lewandrowski K, Lee-Lewandrowski E, et al. Diagnosis of pancreatic cystic neoplasms: a report of the cooperative pancreatic cyst study. Gastroenterology 2004;126:1330-1336.

3. Hill JS, McPhee JT, McDade TP, et al. Pancreatic neuroendocrine tumors: the impact of surgical resection on survival. Cancer 2009;115:741751. 

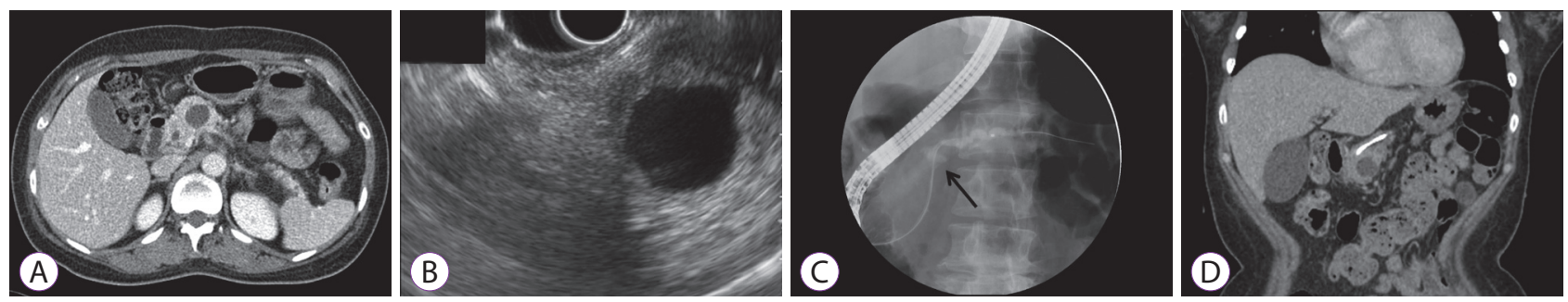

Fig. 1. Imaging modalities for the pancreatic cystic lesion. (A) Abdominal computed tomography (CT) shows a well-defined cystic lesion measuring $2.1 \mathrm{~cm}$ at the neck of the pancreas, connected to the pancreatic duct. (B) Endoscopic ultrasound demonstrates an anechoic cystic lesion without solid content. (C) Pancreatography shows a connection of the cystic lesion (arrow) to the main pancreatic duct. (D) Follow-up CT shows no interval change in the size of the cystic lesion despite the long-lasting plastic stent.
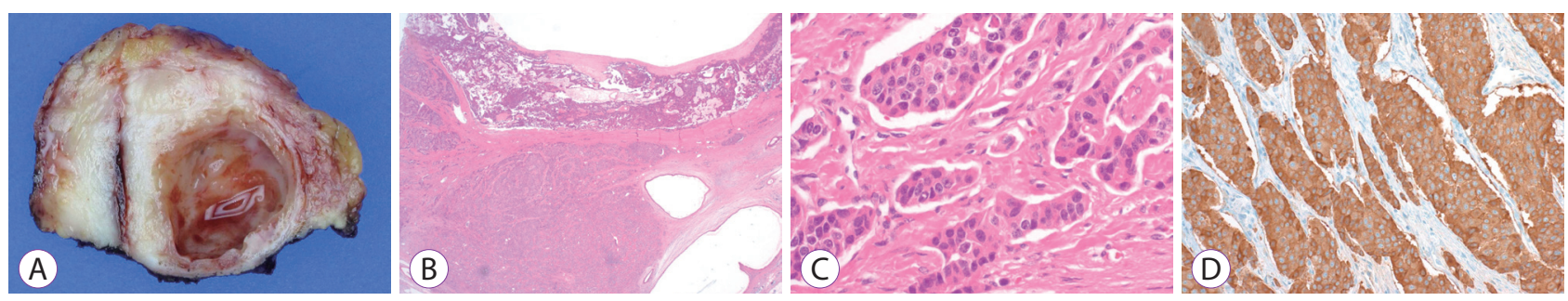

Fig. 2. Histopathologic examination of the resected pancreatic cystic lesion. (A) Gross examination shows a unilocular cyst containing serosanguineous fluid. (B-D) The surrounding solid component diagnosed with a grade 2 neuroendocrine tumor $(B, \times 12$, hematoxylin and eosin $[H \& E] ; C, \times 400, H \& E ; D, \times 200$, synaptophysin). 